\title{
The Responsibility of Healthcare Institutions to Protect Global Health Security
}

Kumanan Wilson, Christopher McDougall and Alan Forster

$\mathbf{N}$

ew public threats that can rapidly cross borders are continuing to challenge global health security (World Health Organization [WHO] 2007). To protect the world from these threats, unprecedented levels of co-operation are required (Wilson et al. 2008). At the international level, the response to this challenge led to the approval of revised International Health Regulations (IHR) (WHO 2005). This unanimously approved document outlines how countries are to prepare for and respond to public health emergencies of international concern in a manner that does not unnecessarily impact on travel and trade.

At first glance, the IHR may appear to be primarily concerned with global health policy and of little concern to healthcare institutions. However, the IHR are cognizant of the critical role played by local responders in detecting and controlling emerging health threats. As important as co-operation between countries is to prepare for public health emergencies, the response at the local level is perhaps more important. Public health emergencies initially present as isolated cases or a cluster of cases at the local level. These may be detected by local public health officials or be identified in healthcare institutions. In order to effectively protect against the rapid spread of these threats, local health officials must be able to detect these threats and have the capacity to introduce measures to control their spread. Consequently, the regulations provide recommendations that are highly relevant to local public health officials and healthcare institutions.

On June 15, 2007, the regulations became binding international

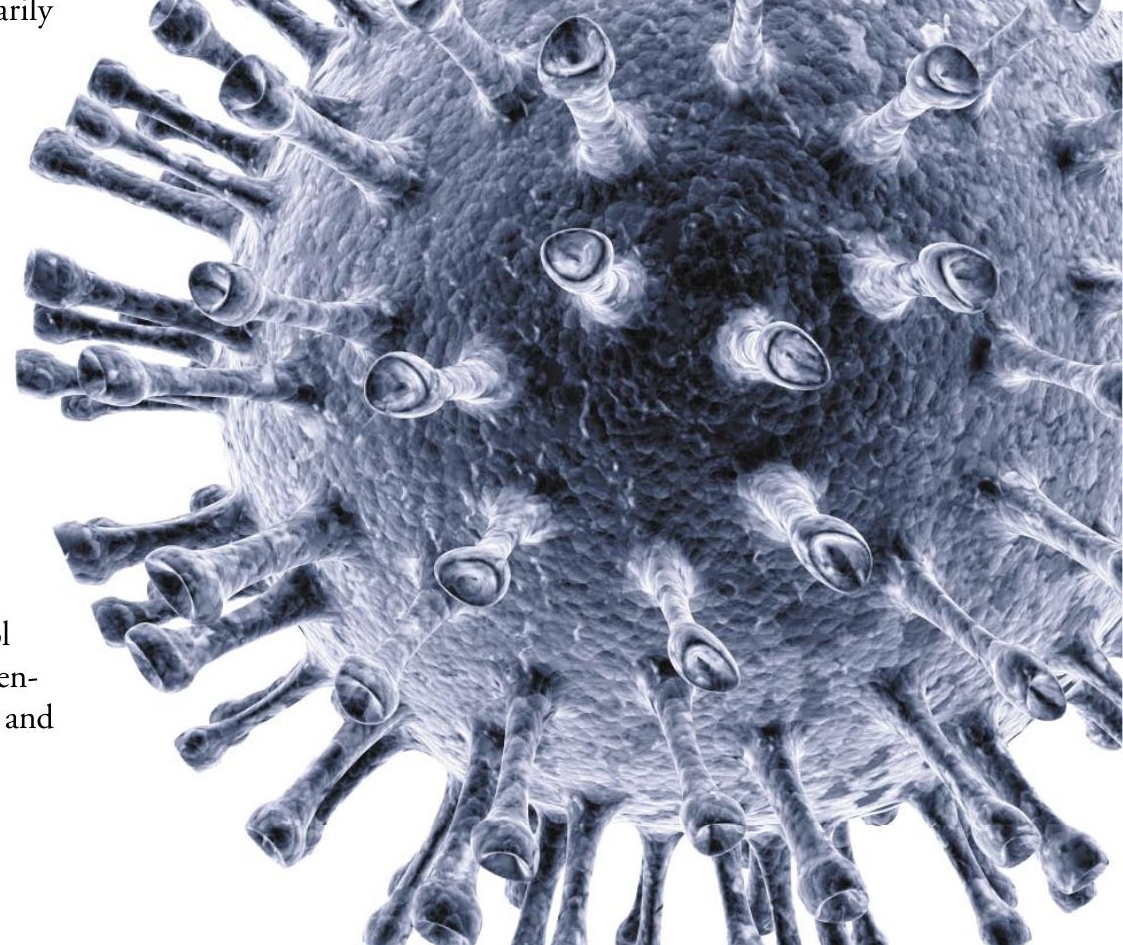


law. And within five years of their establishment, countries are expected to be compliant with their requirements. Healthcare administrators will have to be aware of the expectations of their institutions to both prepare for and respond to a public health emergency. They will also need to be aware of the assistance they should expect to receive from their national governments as per the requirements outlined in the new regulations.

\section{What Are the IHR?}

The outbreak of severe acute respiratory syndrome (SARS) in 2002-2003 demonstrated the complexities and importance of effectively managing a health crisis of global scope (Fidler 2003). In doing so, it also identified numerous points of contention and issues that needed resolution: At what point should countries inform others countries of a potential health threat? Under what circumstances is it acceptable for a country to close its borders because of fears of international spread of disease? What role should WHO play in controlling the international spread of public health threats? To a large extent, the revisions to the IHR were intended to provide guidance on these issues. The previous version of the regulations had numerous limitations, not the least of which was that it pertained to only three diseases (WHO 1983). The revised version of the regulations, designed for the realities of the modern world, needed to be sufficiently flexible to extend beyond these diseases, or any list of diseases, since new threats are regularly presenting themselves. Revised regulations also had to be applicable to non-infectious threats that could cross international borders, for example, chemical agents or radio-nuclear material.

The new IHR accommodates these possibilities by pioneering the concept of a "public health emergency of international concern" (WHO 2005). Any event that meets any two of the following will be considered a potential public health emergency of international concern and will have to be reported to WHO:

- The public health impact of the event is serious.

- The event is unusual or unexpected.

- There is a significant risk of international spread.

- There is a significant risk of international travel or trade restrictions.

Upon receiving notification of an event that meets these criteria, obtaining additional information and conducting the appropriate consultations, $\mathrm{WHO}$ will then determine if the event is to be formally declared a public health emergency of international concern. If so, WHO will then recommend appropriate measures to limit the spread of the emergency, for example, to not travel to an affected region. In making these recommendations, WHO will attempt to do so in a manner that does not unnecessarily interfere with international travel and trade.
In addition to describing an explicit mechanism by which international public health emergencies are to be identified, reported and responded to, the new IHR also provide clear instructions for countries to develop the capacity to detect, report and respond to these emergencies. These include developing capacity to conduct surveillance, to transmit information and to provide an initial response to the emergency. Countries are expected to have these capacities in place within five years of the regulations coming into force. The core capacities stated in the IHR are divided into local, intermediate and national capacities. The requirements, outlined in Annex 1 of the agreement, are presented in Table 1 (WHO 2005).

\section{The Responsibilities of Healthcare Institutions under the New International Rules}

The IHR are an agreement approved by national governments. It is the responsibility of federal or national governments to ensure compliance with the agreements. However, many of the aspects of the regulations are focused very much at the local level, reflecting recognition that the local level is the key to early detection and response - and perhaps preventing the emergence of a larger epidemic.

\section{Many potential public health emergencies of international concern may first become evident at the hospital level.}

As the SARS outbreak made evident, the first response to a public health emergency may often be at the level of the hospital (National Advisory Committee on SARS and Public Health 2003). Healthcare institutions are often where new diseases cluster and are recognized to be abnormal in prevalence. Such recognition can be difficult at the community level because multiple different care providers in geographically separate regions may be seeing only isolated cases. Healthcare institutions are also where emerging infections can initially spread, through nosocomial transmission. Many potential public health emergencies of international concern may therefore first become evident at the hospital level. Under the IHR, it will be the healthcare institution's responsibility to make this recognition and communicate it to public health officials. Therefore, many of the core capacities that the IHR require countries to have in place are directly relevant to healthcare institutions and fall within the local capacity classification.

First, healthcare institutions must develop the capacity to "detect events involving disease or death above expected levels for the particular time and place in all areas within the territory of the State Party" (WHO 2005; see Table 1). To accomplish this objective, it is evident that healthcare institutions will need 
Table 1. Core capacity requirements for surveillance and response

\section{At the local community level and/or primary public health response level} The capacities:

(a) to detect events involving disease or death above expected levels for the particular time and place in all areas within the territory of the State Party; and

(b) to report all available essential information immediately to the appropriate level of healthcare response. At the community level, reporting shall be to local community health-care institutions or the appropriate health personnel. At the primary public health response level, reporting shall be to the intermediate or national response level, depending on organizational structures. For the purposes of this Annex, essential information includes the following: clinical descriptions, laboratory results, sources and type of risk, numbers of human cases and deaths, conditions affecting the spread of the disease and the health measures employed; and

(c) to implement preliminary control measures immediately.

\section{At the intermediate public health response levels}

The capacities:

(a) to confirm the status of reported events and to support or implement additional control measures; and

(b) to assess reported events immediately and, if found urgent, to report all essential information to the national level. For the purposes of this Annex, the criteria for urgent events include serious public health impact and/or unusual or unexpected nature with high potential for spread.

\section{At the national level}

Assessment and notification. The capacities:

(a) to assess all reports of urgent events within 48 hours; and

(b) to notify WHO immediately through the National IHR Focal Point when the assessment indicates the event is notifiable pursuant to paragraph 1 of Article 6 and Annex 2 and to inform WHO as required pursuant to Article 7 and paragraph 2 of Article 9.

\section{Public health response. The capacities:}

(a) to determine rapidly the control measures required to prevent domestic and international spread;

(b) to provide support through specialized staff, laboratory analysis of samples (domestically or through collaborating centres) and logistical assistance (e.g. equipment, supplies and transport);

(c) to provide on-site assistance as required to supplement local investigations; (d) to provide a direct operational link with senior health and other officials to approve rapidly and implement containment and control measures;

(e) to provide direct liaison with other relevant government ministries;

(f) to provide, by the most efficient means of communication available, links with hospitals, clinics, airports, ports, ground crossings, laboratories and other key operational areas for the dissemination of information and recommendations received from WHO regarding events in the State Party's own territory and in the territories of other States Parties;

(g) to establish, operate and maintain a national public health emergency response plan, including the creation of multidisciplinary/multisectoral teams to respond to events that may constitute a public health emergency of international concern; and (h) to provide the foregoing on a 24-hour basis

Source: Reproduced with permission from Annex 1 of the Revision of the International Health Regulations (World Health Organization 2005). systems to accumulate and organize patient information on diseases and identify changes in disease prevalence to detect an emerging problem. This will require accurate coding and classification of diseases as well as sophisticated informatics systems that can aggregate and continuously monitor data on diseases. These systems also need to be linked to centralized public health systems and must therefore be compatible with other health informatics systems. Most hospitals do not have sophisticated electronic health records, so they would have to rely on administrative data systems such as the hospital discharge abstract. There are two reasons why these are inadequate to meet the requirements outlined in the regulations. There are significant lags between patient encounters and documentation in the administrative data systems currently used for tracking diagnoses. Coupling this delay with the probability of inaccurate coding within administrative data systems further illustrates the limitations with using existing hospital information systems (Campbell et al. 2001).

Even if hospitals do have electronic health records, they will also require the infrastructure to perform the necessary analyses. Such investigations will have to be able to identify small trends in the prevalence of disease laboratory abnormalities or a cluster of symptoms, which might signify an outbreak early enough to act. Healthcare institutions will therefore require infrastructure such as a data warehouse, which is a central repository of health information, to enable analyses of integrated information (Bates et al. 1999). They also must have a cadre of analysts and statisticians who are familiar with health data and can perform and interpret the analyses. Hospital information systems will also have to be fully integrated with broader community health information infrastructures so that local events can be compared with broader population ones.

Second, healthcare institutions will have to be able "to report all available essential information immediately to the appropriate level of healthcare response. At the community level, reporting shall be to local community health-care institutions or the appropriate health personnel. At the primary public health 
response level, reporting shall be to the intermediate or national response level, depending on organizational structures" (WHO 2005; see Table 1). This requires healthcare institutions to be closely integrated with local public health units. Relationships must not simply be informal but formally outlined with clear protocols so that the transfer of information is not discretionary and will occur despite perceptions of harm done to an institution of the disclosure of such information. Having integrated health information systems that will flow to public officials automatically will be an important step. Obviously and critically, privacy issues related to transfer information need to be addressed in advance of developing such systems.

\section{It would appear that for the federal government to assist healthcare institutions in meeting the IHR core capacity requirements, it would have to do so with provincial permission.}

Finally, institutions will need to develop the capacity "to implement preliminary control measures immediately" (WHO 2005). Healthcare institutions are expected to be well equipped to address public health emergencies. Most healthcare institutions have taken important steps in this regard through the development of pandemic influenza plans. The IHR do not pertain only to pandemic influenza or other infectious pandemics but, rather, to all public health emergencies that pose a potential international threat. Therefore, healthcare institutions will need to build on their existing pandemic plans and move toward an all-hazards approach.

Nevertheless, this requirement will be almost impossible for hospitals to meet without significant investments in their capacity. The current bed situation in acute care hospitals is such that hospitals are often at, or above, full capacity. This often results in admitted patients waiting in the emergency room for a bed (Forster et al. 2003). In addition, most hospitals and emergency rooms have a limited number of private rooms, which are commonly used for isolating patients with potentially communicable diseases. Finally, the knowledge, attitude and behaviours of most healthcare workers toward effective practices for reducing the risk of communicable disease transmission may not be sufficient. For example, compliance rates with hand-washing practices are often less than $60 \%$ for all hospital workers and much lower for physicians (Gawande 2004). Thus, given the current environment in hospitals, it will be difficult to ensure that emergency control measures that meet IHR requirements are introduced.

In summary, to meet the requirements of the revised IHR, hospitals and healthcare centres should do the following:

1. Have in place informatics systems that can aggregate and monitor data

2. Have mechanisms by which this information can easily flow to public health and be compatible with other health information these centres receive

3. Develop protocols for information sharing with local public health officials

4. Address potential privacy issues

5. Have all-hazards protocols to deal with infectious and noninfectious public health emergencies

\section{What Assistance Should Healthcare Institutions Expect to Receive?}

The regulations place significant new expectations on healthcare institutions. However, the regulations do not expect healthcare institutions to fulfill these expectations without assistance. Recognizing that the local response is essential to preventing the spread of public health emergencies, the regulations require the national level of government to provide the following (WHO 2005; see Table1):

- Support through specialized staff, laboratory analyses of samples (domestically or through collaborating centres) and logistical assistance (e.g., equipment, supplies and transport)

- On-site assistance as required to supplement local investigations

- By the most efficient means of communication available, links with hospitals, clinics, airports, ports, ground crossings, laboratories and other key operational areas for the dissemination of information and recommendations received from WHO regarding events in the State Party's own territory and in the territories of other States Parties

Healthcare institutions should therefore be able to request assistance in detecting and evaluating the emergence of potential public health emergencies. Hospitals should also be able to call on higher levels of government for assistance in identifying and communicating information on a possible public health emergency of international concern.

However, the division of powers in Canada's constitution could provide some impediments to receiving assistance from the national level of government (Wilson and MacLennan 2005). Logically, the local level could be expected to receive assistance from the Public Health Agency of Canada. However, under the constitution, healthcare is a provincial responsibility and public health is a shared responsibility. While the federal government has some jurisdiction over issues relating to responding to public health emergencies, in the case of 
emergencies limited to a single province, the federal government would have to ask permission from the provincial government to intervene. Similarly, it would appear that for the federal government to assist healthcare institutions in meeting the IHR core capacity requirements, it would have to do so with provincial permission. A potential scenario in how this could occur would involve the federal government flowing money to the provinces, which would then provide the requisite assistance to local public health and healthcare institutions.

\section{Conclusion}

The SARS outbreak demonstrated the havoc that public health emergencies can cause at the local, provincial, national and international levels and the link between all these levels of responses. A rethink of approaches to similar emergencies that may present themselves in the future recognized the importance of addressing these emergencies at their earliest stages. The local health response was found to be a key component of the new approach.

Healthcare institutions should immediately initiate implementing the necessary measures to be compliant with the new regulations. Failure to do so by 2012 could mean that Canada could be in violation of international law. Hospitals and healthcare centres will need to make significant investments to ensure that they can make these investments within the next five years. While these investments are necessary for the purposes of the IHR, they may have benefits beyond them. Meeting the IHR requirements will create a more integrated health system - not one artificially divided between public health and healthcare - and hospital informatics systems that can provide real-time information on disease burdens. Better information systems and the capacity to analyze hospital performance data will lead to better-run hospitals. Improved infrastructure with enhanced capacity to isolate patients, coupled with greater adherence to infection-prevention behaviours by hospital workers, will reduce the incidence of hospital-acquired infections. Thus, there are compelling reasons beyond the IHR to invest in these infrastructures, and it should therefore be a priority of all levels of government to make this occur. HQ

\section{Acknowledgements}

This article is based in part on information emerging from a workshop examining the implementation of the IHR in decentralized states (www.ihrfederalism.com). This workshop was supported by grants from the Canadian Institutes of Health Research, the Public Health Agency of Canada, the Canadian International Development Agency and the International Development Research Centre. We acknowledge the contributions of all of those who participated in this workshop.
Kumanan Wilson is the recipient of the Canada research chair in public health policy. Alan Forster is the recipient of an Ontario Ministry of Health and Long-term Care career scientist award.

\section{References}

Bates, D.W., E. Pappius, G.J. Kuperman, D. Sittig, H. Burstin, D. Fairchild, T.A. Brennan and J.M. Teich. 1999. "Using Information Systems to Measure and Improve Quality. International Journal of Medical Informatics 53(2-3):115-24.

Campbell, S.E., M.K. Campbell, J.M. Grimshaw and A.E. Walker. 2001. "A Systematic Review of Discharge Coding Accuracy." Journal of Public Health Medicine 23(3): 205-11.

Fidler, D.P. 2003. "SARS: Political Pathology of the First PostWestphalian Pathogen." Journal of Law, Medicine and Ethics 31(4): 485-505.

Forster, A.J., I. Stiell, G. Wells, A.J. Lee and C. van Walraven. 2003. "The Effect of Hospital Occupancy on Emergency Department Length of Stay and Patient Disposition." Academic Emergency Medicine 10(2): $127-33$.

Gawande, A. 2004. "On washing hands." New England Journal of Medicine 350(13): 1283-6.

National Advisory Committee on SARS and Public Health. 2003. "SARS in Canada: Anatomy of an Outbreak." In National Advisory Committee on SARS and Public Health, Learning from SARS: Renewal of Public Health in Canada. Ottawa, ON: Health Canada.

Wilson, K. and C. MacLennan. 2005. "Federalism and Public Health Law: Opportunities and Unanswered Questions." Health Law Review 14: 3-13.

Wilson, K., B. von Tigerstrom and C. McDougall. 2008. "Protecting Global Health Security through the International Health Regulations: Requirements and Challenges. Canadian Medical Association Journal 179(1): 44-48.

World Health Organization. 1983. International Health Regulations (3rd ed.). Geneva, Switzerland: Author.

World Health Organization. 2007. The World Health Report 2007. A Safer Future. Global Public Health Security in the 21st Century. Geneva, Switzerland: Author.

World Health Organization, Fifty-Eighth World Health Assembly. 2005. Revision of the International Health Regulations. Geneva, Switzerland: Author. Retrieved November 8, 2008. <www.who.int/ gb/ebwha/pdf_files/WHA58/A58_4-en.pdfs.

\section{About the Authors \\ Kumanan Wilson, MD, MSc, FRCP(C), is with the Ottawa Health Research Institute, Department of Medicine, University of Ottawa, Ottawa, Ontario, and the Joint Centre for Bioethics at the University of Toronto, Toronto, Ontario.}

Christopher McDougall, MA, is with the Joint Centre for Bioethics at the University of Toronto.

Alan Forster, MD, MSc, FRCP(C), is with the Ottawa Health Research Institute, Department of Medicine, University of Ottawa. 